\title{
Nonlinear Optical Functions in Crystalline and Amorphous Silicon-on-Insulator Nanowires
}

\author{
R. Baets ${ }^{1,2^{*}}$, B. Kuyken ${ }^{1,2}$, X. Liu $^{3}$, S. Clemmen ${ }^{4, \#}$, S. Selvaraja ${ }^{1,2}$, W. Bogaerts ${ }^{1,2}$, D. Van Thourhout ${ }^{1,2}$, H. Ji ${ }^{7}$, \\ H. $\mathrm{Hu}^{7}$, M. Pu ${ }^{7}$, M. Galili ${ }^{7}$, P. Jeppesen ${ }^{7}$, L.K. Oxenløwe ${ }^{7}$, R. M. Osgood, Jr. ${ }^{3}$, P. Emplit ${ }^{5}$, S. Massar ${ }^{4}$, Y.A. \\ Vlasov $^{6}$, W. M. J. Green ${ }^{6}$, G. Roelkens ${ }^{1,2}$ \\ ${ }^{1}$ Photonics Research Group, Department of Information Technology, Ghent University - imec, Ghent, Belgium \\ ${ }^{2}$ Center for Nano- and Biophotonics (NB-Photonics), Ghent University, Ghent, Belgium. \\ ${ }^{3}$ Department of Electrical Engineering, Columbia University, New York, NY 10027, USA \\ ${ }^{4}$ Laboratoire d'Information Quantique (LIQ), Université Libre de Bruxelles (U. L. B.), Bruxelles B-1050, Belgium \\ ${ }^{5}$ Service OPERA-Photonique, Université libre de Bruxelles (U.L.B.), Bruxelles B-1050, Belgium \\ ${ }^{6}$ IBM T. J. Watson Research Center, Yorktown Heights, NY 10598, USA \\ ${ }^{7}$ DTU Fotonik, Technical University of Denmark, DK-2800 Kgs. Lyngby, Denmark \\ "now at School of Applied and Engineering Physics, Cornell University, Ithaca, New York 14853, USA \\ *Roel.Baets@UGent.be
}

\begin{abstract}
Silicon-on-Insulator nanowires provide an excellent platform for nonlinear optical functions in spite of the two-photon absorption at telecom wavelengths. Work on both crystalline and amorphous silicon nanowires is reviewed, in the wavelength range of 1.5 to $2.5 \mu \mathrm{m}$. OCIS codes: (190.4380) Four wave mixing; (130.4310) Nonlinear integrated optics
\end{abstract}

\section{Introduction}

The high nonlinear index of silicon, combined with the high confinement in silicon-on-insulator (SOI) nanowires due to its high linear index have made the silicon-on-insulator technology an excellent platform for nonlinear optical functions and all-optical signal processing at low power. However, at telecom wavelengths crystalline silicon suffers, as a result of the two-photon absorption process (TPA), from extensive nonlinear absorption. This translates in a poor nonlinear figure of merit for crystalline silicon, defined as the ratio of the nonlinear refraction to the nonlinear absorption.

The TPA-problem is obviously absent for photon energies below half the bandgap of silicon and therefore SOInanowires are ideally suited for nonlinear optical functions in the wavelength range beyond $2.2 \mu \mathrm{m}$. In the first part of this paper our recent work in this field is summarized.

At telecom wavelengths one can mitigate the TPA-problem by using silicon only for confinement and by using a cladding material as nonlinear material. This is the approach taken in Silicon-Organic-Hybrid ( $\mathrm{SOH}$ ) waveguides, whereby a strongly nonlinear optical polymer is filling the gap of a slotted waveguide. With this approach advanced all-optical signal processing functions have been demonstrated [1,2]. A technological problem associated with this is that it is very challenging to make slotted waveguides with low linear loss.

Another approach is to replace crystalline silicon by amorphous silicon as the nanowire material. This replacement has little impact on the linear properties but improves the nonlinear figure of merit considerably. In the second part of this paper our work on nonlinear optical functions in amorphous silicon is summarized.

\section{Crystalline silicon-on-insulator nanowires at wavelengths beyond $2 \mu \mathrm{m}$.}

Not only is a silicon nanowire a unique waveguide for strong nonlinear optical effects, but the high index contrast also allows to engineer the dispersion - by choosing the dimensions of the waveguide cross-section appropriately so as to achieve unique phase matching properties for four-wave mixing processes in the short-wave infrared [3]. This approach has allowed to demonstrate strong modulation instability as well as very high parametric gain (> 40 $\mathrm{dB}$ gain in a $2 \mathrm{~cm}$ long waveguide) [4], supercontinuum generation [5,6], and wavelength conversion from $2.45 \mu \mathrm{m}$ down to telecom wavelengths and vice versa [7]. It has also allowed to demonstrate a widely tunable Optical Parametric Oscillator by embedding the silicon chip into a fiber ring cavity [8]. 


\section{Hydrogenated amorphous silicon-on-insulators nanowires at telecom wavelengths}

Amorphous hydrogenated silicon (a-Si:H) photonic wires are considered to be a good alternative for the standard crystalline silicon-on-insulator photonic wires. a-Si:H layers can be deposited at relatively low temperatures by means of Plasma Enhanced Chemical Vapour Deposition and thus be back-end deposited on a finished CMOS wafer. Several integrated photonic components based on the amorphous silicon platform, including high Q resonators, Mach-Zehnder interferometers and low-loss $(3.6 \mathrm{~dB} / \mathrm{cm})$ single mode photonic wires have been demonstrated [9].

At telecom wavelengths the nonlinear refractive index turns out to be higher than that of crystalline silicon and its nonlinear absorption is lower, resulting in a figure of merit about 4 times higher than that of c-Si [10]. This has allowed to demonstrate useful nonlinear functions at telecom wavelengths including $26 \mathrm{~dB}$ parametric gain in a 1.1 $\mathrm{cm}$ long waveguide [11,12] and optical waveform sampling at $320 \mathrm{~Gb} / \mathrm{s}$ [13].

An issue with a-Si:H is that it is not stable under high intensity photon illumination [14]. This effect is assumed to be related to the Staebler-Wronski effect in a-Si solar cells. However the amorphous silicon can be fully regenerated by thermal annealing at $200^{\circ} \mathrm{C}$. Work is underway to see whether the material properties of a-Si:H can be optimized with respect to this degradation problem.

\section{References}

[1] T. Vallaitis, S. Bogatscher, L. Alloatti, P. Dumon, R. Baets, M. L. Scimeca, I .Biaggio, F. Diederich, C., W. Freude, and J. Leuthold, “Optical properties of highly nonlinear silicon-organic hybrid (SOH) waveguide geometries,” Opt. Express 17, 17357-17368 (2009).

[2] C. Koos, P. Vorreau, T. Vallaitis, P. Dumon, W. Bogaerts, R. Baets, B. Esembeson, I. Biaggio, T. Michinobu, F. Diederich, W. Freude, and J. Leuthold, “All-optical high-speed signal processing with silicon-organic hybrid slot waveguides,” Nature Photon. 3, $216-219$ (2009).

[3] W.M.J. Green, X. Liu, B. Kuyken, R.M. Osgood, G. Roelkens, R. Baets, Y. Vlasov, Nonlinear silicon nanophotonics for mid-infrared applications, Information Photonics 2011 United States, (2011).

[4] X. Liu, B. Kuyken, G. Roelkens, R. Baets, Y. Vlasov, R.M. Osgood, W.M.J. Green, Mid-infrared broadband modulation instability and 50dB Raman assisted parametric gain in silicon photonic wires, CLEO 2011, United States, (2011).

[5] B. Kuyken, X. Liu, R.M. Osgood, Y. Vlasov, R. Baets, G. Roelkens, W.M.J. Green, Generation of a telecom-to-mid-infrared spanning supercontinuum using silicon-on-insulator wire waveguides, CLEO 2011, United States, (2011).

[6] B. Kuyken, X. Liu, R. M. Osgood Jr., R. Baets, G. Roelkens, W. M.J. Green, Mid-infrared to telecom-band supercontinuum generation in highly nonlinear silicon-on-insulator wire waveguides, Optics Express, 19, p.20172-20181 (2011)

[7] B. Kuyken, X. Liu, R.M. Osgood, Y. Vlasov, G. Roelkens, R. Baets, W.M.J. Green, Frequency conversion of mid-infrared optical signals into the telecom band using nonlinear silicon nanophotonic wires, OFC 2011, United States, (2011).

[8] B. Kuyken, X. Liu, R. M. Osgood Jr., R. Baets, G. Roelkens, W. M. J. Green, Widely Tunable Silicon Mid-Infrared Optical Parametric Oscillator, Group IV Photonics, United Kingdom, (2011).

[9] S. Selvaraja, Erik Sleeckx, Marc Schaekers, W. Bogaerts, D. Van Thourhout, P. Dumon, R. Baets, Low-Loss Amorphous Silicon-OnInsulator Technology for Photonic Integrated Circuitry, Optics Communications 282, 1767-1770 (2009).

[10]B. Kuyken, S. Clemmen, S. Selvaraja, W. Bogaerts, S. Massar, R. Baets, G. Roelkens, Self phase modulation in highly nonlinear hydrogenated amorphous silicon, IEEE Photonics Society Annual Meeting, United States, (2010).

[11]S. Clemmen, B. Kuyken, S. Selvaraja, W. Bogaerts, S. Massar, D. Van Thourhout, Ph. Emplit, R. Baets, G. Roelkens, Unprecedented parametric gain from a CMOS compatible silicon photonic wire, IEEE Photonics Society Annual Meeting 2010, United States, (2010).

[12]B. Kuyken, S. Clemmen, S. Selvaraja, W. Bogaerts, D. Van Thourhout, Ph. Emplit, S. Massar, G. Roelkens, R. Baets, On-chip parametric amplification with $26.5 \mathrm{~dB}$ gain at telecommunication wavelengths using CMOS-compatible hydrogenated amorphous silicon waveguides, Optics Letters, 36, p.552-554 (2011).

[13]H. Ji, H. Hu, M. Pu, M. Galili, P. Jeppesen, B. Kuyken, S. Selvaraja, G. Morthier, R. Baets, L.K. Oxenløwe, Optical Waveform Sampling of a 320 Gbit/s Serial Data Signal using a Hydrogenated Amorphous Silicon Waveguide, European Conference and exhibition on Optical Communication, Switzerland, (2011).

[14]B. Kuyken, X. Liu, S. Clemmen, S. Selvaraja, W. Bogaerts, D. Van Thourhout, R. M. Osgood, P. Emplit, S. Massar, Y. A. Vlasov, W. M. J. Green, G. Roelkens, R. Baets, Four-Wave-Mixing Gain and All-optical Signal Processing in Silicon Nanowires, European Conference and exhibition on Optical Communication, Switzerland, (2011). 